\title{
Natural Antibiofilm Agents and the Need for Antibiofilm Drug Leads
}

\author{
${ }^{1}$ Hazniza Adnan*, ${ }^{2}$ Veronique Seidel \& ${ }^{2}$ Nicholas P. Tucker \\ ${ }^{1}$ Biotechnology and Nanotechnology Research Centre, \\ Malaysian Agricultural Research and Development Institute, Malaysia \\ ${ }^{2}$ Strathclyde Institute of Pharmacy and Biomedical Sciences, University of Strathclyde, Glasgow, U.K. \\ *Corresponding author: hazniza@mardi.gov.my
}

\begin{abstract}
Plant with medicinal property contains different types of bioactive compounds and these compounds are effective against certain diseases. Biofilm infection is a disease caused by biofilm which is associated with the respiratory and gastrointestinal infections. Biofilm-producing bacteria as such Pseudomonas aeruginosa strain PA14 is a serious treat in hospitals. The bacterium possesses many virulent factors that contributed to its pathogenesis and resistance thus caused the inhibition and removal of its biofilms a difficult task. This study therefore attempts to identify natural antibiofilm agents against the $P$. aeruginosa PA14 biofilms. The activity of plant extracts and subsequently purified compounds was evaluated using a stepwise separation process called bioassay-guided fractionation. The fractionation process involved the use of chromatographic techniques and compounds were identified using NMR, GC-MS and LC-MS. A total of four bioactive fractions namely (E333F1S1), (E341), (HA6) and (M338B) have been discovered with antibiofilm activity and were selected for further investigation. The active fraction from Ribes nigrum leaf (E333F1S1) contained mixtures of alkanes while active extract of Sambucus nigra flower (E341) contained a mixture of ursolic acid and oleanolic acid. The LC-MS analysis on Coriandrum sativum seeds (HA6) revealed the presence mixture of oxygenated monoterpenes, while another active fraction of $C$. sativum seeds (M338B) showed the presence of carboxylic acid, carboxylate, tetraone, glycerol, carbohydrate and fatty acids. Thus, the discovery of potential bioactive compounds from this study could be further investigated as antibiofilm drug leads to battle the infections by $P$. aeruginosa PA14 biofilm in the near future.
\end{abstract}

Keywords Pseudomonas aeruginosa strain PA14, biofilm, bioassay-guided fractionation, NMR, GC-MS and LCMS

\section{INTRODUCTION}

Tremendous plant extracts have been used in traditional folk medicine and many has been welldocumented for the treatment of certain diseases. Plant natural products have demonstrated a range of biological effect including antimicrobial [1] and antibiofilm [2] activity. Many drugs currently on the market are either plant natural products or are based on natural product templates. These include morphine from opium, quinine from cinchona, artemisinin, salicylic acid, rivastigmine, digitalis glycosides and reserpine [3]. Many of the bioactive phytochemicals in such active plant natural products however remain to be identified. Plant natural product could provide novel natural antibiofilm agents to fulfill the urgent need as therapies for Pseudomonas-related infections.

Pseudomonas aeruginosa is a Gram-negative bacterium and rod shaped with a single polar flagellum. $P$. aeruginosa strain PA14 is a nosocomial pathogen and the primary cause of death in immunecompromised patients. P. aeruginosa PA14 can cause various skin infections both localised and diffused, and tendency to colonise the surfaces in a biofilm form. $P$. aeruginosa PA14 is the most prevalent infective pathogen and possessed a large number of virulence factors and antimicrobial genes [4]. The membrane of $P$. aeruginosa PA14 acts as a permeability barrier and makes the cells impervious to therapeutic concentration such as antibiotics.

The study on the resistance of $P$. aeruginosa PA14 biofilms has gained a wide interest to explore indepth. Plant-derived compounds consist a wide range of pharmacological properties, including antioxidant, immunostimulant, anticoagulant, hypocholesterolemic and antimicrobial activity [5], antiviral [6] and antibiofilms [7]. Thus, those bioactive compounds with antibiofilm property could be a great 
choice to inhibit the P. aeruginosa PA14 biofilms and could also provide templates for new antibiofilm drug discovery.

Bioassay guided fractionation method can be used to isolate compounds with antibiofilm activity in plant extracts. The chemical diversity, chirality and various functional groups present in natural products make them an ideal source of new antibiofilm compounds [8]. More than hundreds of plant extracts have been investigated for the presence of active compounds against $P$. aeruginosa PA14 biofilms, however only four extracts from three potential plants were chosen to be presented for this paper.

\section{EXPERIMENTAL}

\section{Plant materials}

The dried plant materials were purchased from a commercial supplier (G. Baldwin and Co., UK) and some are supplied by Dr. Xianmin Chang (Orkney College UHI, Kirkwall, UK). All plant materials were ground into a fine powder using an analytical mill (IKA A11 BASIC, China). Powdered plant materials are packed in a paper envelope and stored in a dry cabinet in the Natural Product Research Laboratories, Strathclyde Institute of Pharmacy and Biomedical Sciences (SIPBS), University of Strathclyde, UK.

\section{Plant extraction and preparation of samples}

Each plant material $(500 \mathrm{~g} \mathrm{DW})$ was extracted using $n$-hexane $(1 \mathrm{~L})$, ethyl acetate $(1 \mathrm{~L})$ and methanol (1 L) for $1 \mathrm{~h}$ at $25^{\circ} \mathrm{C}$ using a sonicator (DECON F5100b, UK). All extracts were filtered using a qualitative filter paper $(125 \mathrm{~mm}$; Fisher UK) and then concentrated under reduced pressure using a rotary evaporator (Buchi R-205, Switzerland) at $40{ }^{\circ} \mathrm{C}$ and later dried under a flow of nitrogen gas. All extracts were kept sealed in glass containers and stored at $-20^{\circ} \mathrm{C}$. Prior to analysis, each sample was weighed and dissolved in dimethyl sulfoxide (DMSO) to obtain a final concentration of $1 \mathrm{mg} / \mathrm{mL}$.

\section{Preparation of $P$. aeruginosa PA14 assay}

The clinical Pseudomonas aeruginosa strain PA14 [9] was obtained from Dr. Nicholas P. Tucker, SIPBS, UK. A loop-full of $P$. aeruginosa PA14 culture from Luria-Bertani (LB) agar was inoculated into $5 \mathrm{~mL}$ of sterilised LB broth and left in a shaking incubator at $37{ }^{\circ} \mathrm{C}$ overnight. Later, $100 \mu \mathrm{L}$ of overnight culture was then inoculated into $50 \mathrm{ml}$ of sterilised LB broth and incubated with shaking at $37^{\circ} \mathrm{C}$. The assay was prepared by inoculating $25 \mu \mathrm{L}$ of fresh $P$. aeruginosa PA14 working culture into $50 \mathrm{ml}$ of M63 medium and was slowly shaken to obtain a homogenised assay.

\section{Antibacterial activity}

The antibacterial activity of each plant extract against $P$. aeruginosa PA14 was measured using a disc diffusion method. Briefly, $200 \mu \mathrm{L}$ of fresh $P$. aeruginosa PA14 cultures were pipetted and spread on Mueller-Hinton $(\mathrm{MH})$ agar to produce a uniform $P$. aeruginosa PA14 lawn. The $P$. aeruginosa PA14 lawn was then embedded with sterilised filter discs $(6 \mathrm{~mm}$ diameter) and $10 \mu \mathrm{L}$ of each sample $(1 \mathrm{mg} / \mathrm{mL})$ was pipetted onto each disc and incubated overnight at $37^{\circ} \mathrm{C}$. Gentamicin $(1 \mathrm{mg} / \mathrm{mL})$ was used as positive control (Control), while DMSO was used as a negative control. The antibacterial activity was shown by the presence of clear zones of inhibition surrounding the disc and was measured as diameter $(\varnothing, \mathrm{mm})$ of inhibition zone.

\section{Antibiofilm activity}

The antibiofilm activity of $P$. aeruginosa PA14 was quantified using high throughput microtitre plate method [10]. The analysis was earlier designed in the microplate reader software (Microplate reader Spectra Max190, USA). The percentage (\%) of biofilm inhibition for P. aeruginosa PA14 was calculated as below.

Biofilm inhibiton, BFI $(\%)=[1-((\mathrm{T}-\mathrm{BE}) / \mathrm{Cr})] \times 100$ 
Where;

$\mathrm{B}_{\mathrm{E}}$ : blank (ethanol)

T: treatment ( $P$. aeruginosa PA14 assay with sample)

$\mathrm{Ct}$ : treatment control (enriched M63 media with sample)

$\mathrm{Cn}$ : negative control (P. aeruginosa PA14 assay with DMSO)

Cr: robust control $([(\mathrm{Ct}-\mathrm{BE})+(\mathrm{Cn}-\mathrm{BE})])$

\section{Isolation and identification of active compounds}

The activity of samples (e.g. plant extracts and subsequently purified compounds) against $P$. aeruginosa PA14 biofilm was isolated using a stepwise separation process called bioassay-guided fractionation method. Samples showing more than $50 \%$ biofilm inhibition (BFI) were further investigated for the presence of active compounds. Fractionation and isolation for active compounds involved the use of chromatographic techniques and detection of the active compounds were identified using NMR, GC-MS and LC-MS.

\section{RESULTS AND DISCUSSION}

\section{Antibacterial activity}

The antibacterial activity of four selected plant extracts (Figure 1) showed that (H338) is the most active extract in inhibiting the growth of $P$. aeruginosa PA14 while (E341) is the least active. The hexane (H338) and methanol (M338) extracts of Coriandrum sativum seeds inhibited the growth of $P$. aeruginosa PA14 at the value of 9.7 and $8.0 \mathrm{~mm}$, respectively. Whereas, the ethyl acetate extracts of Ribes nigrum leaves (E333) and Sambucus nigra flowers (E341) showed antibacterial activity slightly lower at the value of 9.0 and $7.7 \mathrm{~mm}$, respectively.

The $C$. sativum extracts have shown strong antibacterial activity against $P$. aeruginosa PA14. Compounds such as monoterpene alcohols [11] in C. sativum have shown strong antimicrobial activity against Staphylococcus aureus [12] while the essential oil of $C$. sativum has shown strong activity against Campylobacter jejuni [13], Staphylococcus and Eschericia spp. [14]. The plant extracts however showed weak antibacterial activity in comparison to gentamicin. A reason for this could be the ability of $P$. aeruginosa PA14 to reverse between motile and sessile states depending on a multitude of physiological changes [15]. The physiological changes have been displayed by the swarming motility of $P$. aeruginosa PA14 when tested against certain samples (unpublished data). A weak antibacterial activity was also reported on $P$. fluorescens which displayed the least antibacterial activity against a number of extracts from herbs and spices [16].

\section{Antibiofilm activity}

The $n$-hexane (H338) and the methanol extracts (M338) of C. sativum seeds showed a high antibiofilm activity at the values of 69.2 and $63.3 \%$ BFI, respectively (Figure 2). C. sativum is well known for its antibacterial activity [17]. The only report on antibiofilm activity has been for the essential oil of fresh $C$. sativum against Candida albicans biofilm [18]. To date there has been no study on $C$. sativum seeds against the formation of $P$. aeruginosa PA14 biofilm. This warranted further investigation on the active phytochemicals in $C$. sativum seeds. Results also revealed that both ethyl acetate extracts of $R$. nigrum leaves (E333) and $S$. nigra flowers (E341) possessed antibiofilm activity at the values of 68.3 and $59.2 \%$ BFI, respectively (Figure 2). To our knowledge, this is the first report on the antibiofilm activity of $R$. nigrum leaves and $S$. nigra flowers against the formation of $P$. aeruginosa PA14 biofilm, hence supporting further investigation on their active phytochemicals. 


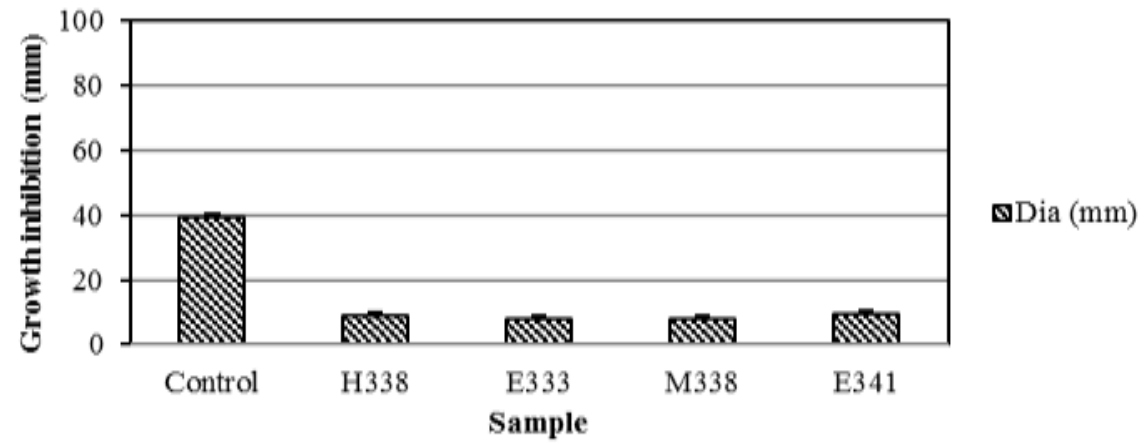

Figure 1 Antibacterial activity of plant extracts against the growth of $P$. aeruginosa PA14 using a disc diffusion assay. Bar charts represent the growth inhibition measured as a diameter (Dia, $\mathrm{mm}$ ) of clear zone exhibited by each sample. Samples coded as H, E and M are referring to $n$-hexane, ethyl acetate and methanol extracts, respectively. Control is gentamicin, a positive control at a concentration of $1 \mathrm{mg} / \mathrm{mL}$. Error bars represent the mean $+\mathrm{SD}$ of three replicates.

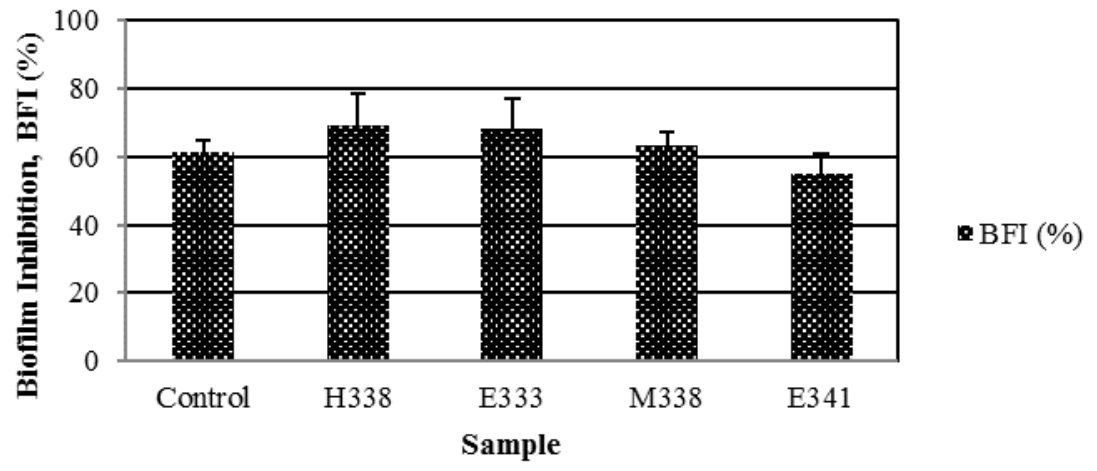

Figure 2 Antibiofilm activity of plant extracts against $P$. aeruginosa PA14 biofilm using high throughput microtitre plate method. Bar charts represent sample with more than $50 \%$ biofilm inhibition (BFI). Samples coded as H, E and $\mathrm{M}$ are referring to $n$-hexane, ethyl acetate and methanol extracts, respectively. Control is gentamicin $(1 \mathrm{mg} / \mathrm{mL}) \mathrm{as}$ a positive control. Error bars represent the mean + SD of six replicates.

\section{Isolation and identification of active compounds}

Figure 3 showed the fractionation of the ethyl acetate extract from Ribes nigrum leaf (E333) against $P$. aeruginosa PA14 biofilm with $68.3 \%$ of biofilm inhibition (BFI). The (E333) was fractionated using VLC into eight fractions with the most active fraction, (E333F1) at 54.3\% BFI. Later (E333F1) was fractionated using Sephadex ${ }^{\circledR}$ column and gave three fractions. (HA30F1S1) at 68.5\% BFI was further investigated using ${ }^{1} \mathrm{H}$ NMR and then subjected to GC-MS analysis. Four identified compounds were nnonadecane, 2-methylnonadecane, 2-methylicosane and 2-methyloctacosane. Further fractionation of (HA30F1S1) using silica column gave seven fractions, however none were active (more than $50 \%$ biofilm inhibition, BFI). The fractionation process had to be terminated. 


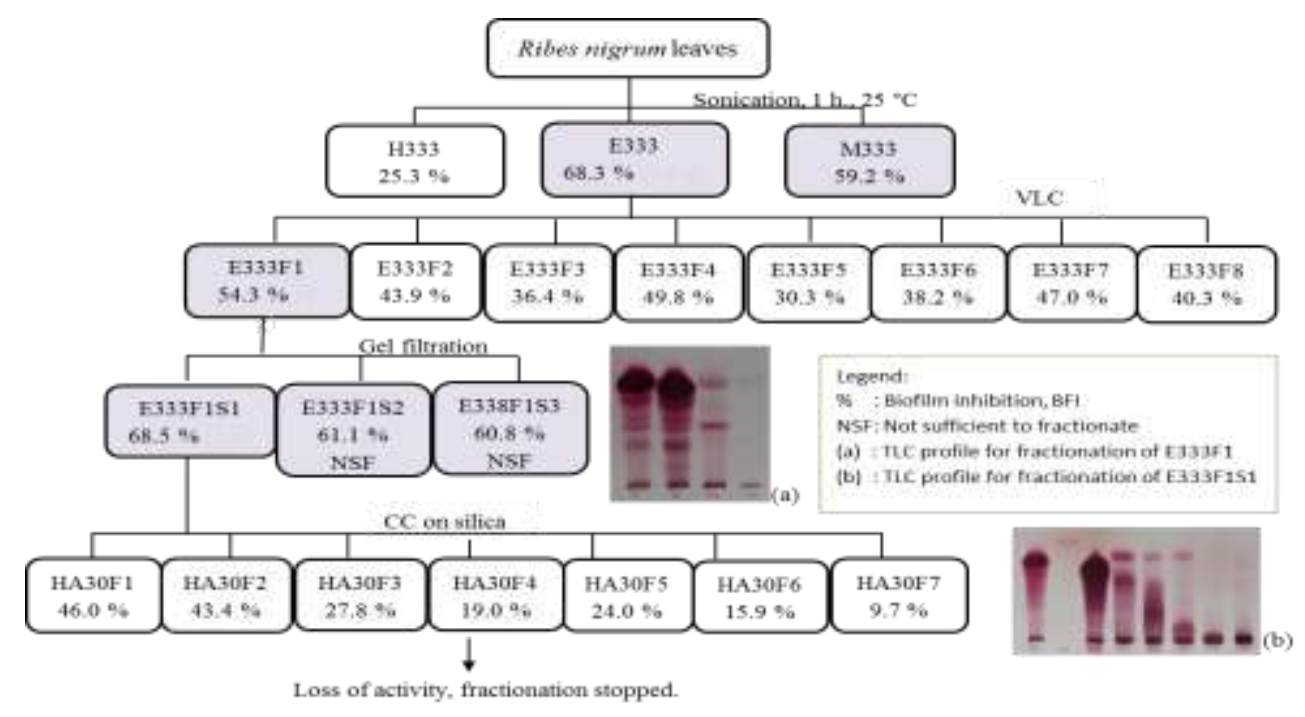

Figure 3 Fractionation scheme of the ethyl acetate extract from Ribes nigrum leaves

Figure 4 presented the HRESI-MS analysis of ethyl acetate extract from Sambucus nigra (E341) in the negative mode indicated a quasi-molecular ion peak [M-H]- at $\mathrm{m} / \mathrm{z} 455.3531$, suggesting a molecular ion $[\mathrm{M}]+$ of 456.3603 and corresponding to the molecular formula of $\mathrm{C} 30 \mathrm{H} 48 \mathrm{O} 3$ (DBE =7). This finding revealed the presence of ursolic and oleanolic acids (Table 1) which is also the first report on the activity of ursolic and oleanolic acids (as a 2:1 mixture) against $P$. aeruginosa PA14 biofilm.

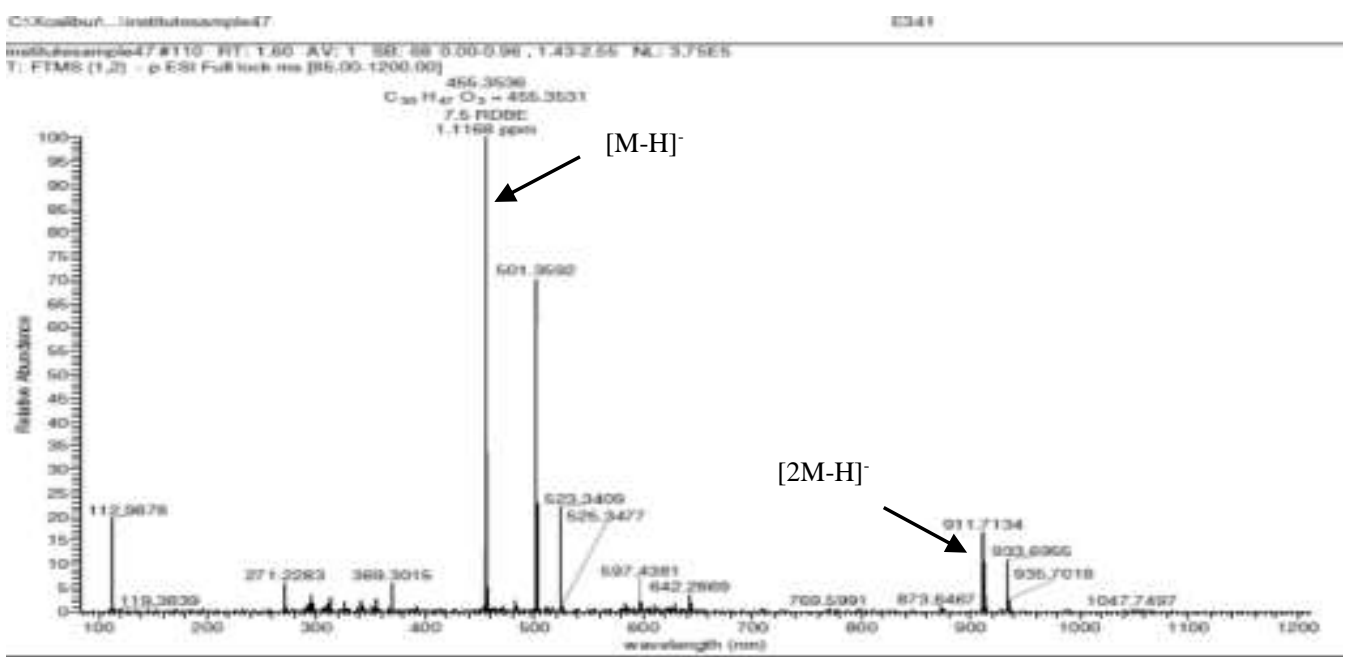

Figure 4 HREI-MS spectrum of Sambucus nigra flower extract (E341)

Figure 5 shows the fractionation scheme obtained for the hexane (H338) and methanol (M338) extracts of $C$. sativum seeds. The separation of phytochemicals was done using bioassay-guided fractionation and isolation methods which are tested against the growth of $P$. aeruginosa PA14 biofilm. Fraction $(\mathrm{H} 338 \mathrm{~F} 3)$ afforded from fractionation of (H338) showed high antibiofilm activity $(57.6 \% \mathrm{BFI})$. Further fractionation of (H338F3) had produced (H338F3S10) and (H338F3S12) which showed strong antibiofilm activity at 60.8 and $67.9 \%$ BFI, respectively. Fractionation of (H338F3S10) afforded (HA6) detected with strong antibiofilm activity at $62.5 \% \mathrm{BFI}$, while (H338F3S12) failed to produce any active fraction with more than $50 \%$ inhibition against $P$. aeruginosa PA14 biofilm. The methanol extract 
(M338) was partitioned into DCM (M338D), n-butanol (M338B) and water (M338W) extracts. Antibiofilm screening showed that all extracts exhibited strong antibiofilm activity. The most active extract was (M338D) with $65.4 \%$ BFI followed by (M338W) and (M338B) at 62.1 and $53.4 \%$ BFI, respectively.

(HA6) was further analysed to detect and identify the active compounds. Both spectra and assignment of ${ }^{1} \mathrm{H}$ and ${ }^{13} \mathrm{C}$ NMR data for (HA6) are presented in Table 1. Later (HA6) was subjected to LC-MS analysis in the positive and negative ESI mode and traces of compounds were identified using MZmine software. Possible metabolites identified in the ESI positive mode (Table 2) include 10-undecenal, linalool oxide (pyranoid and furanoid), 4-ethylcamphor, dodecanal, 2-hexylfuran and caryophyllene oxide. Possible metabolites identified in the ESI negative ionisation mode of (HA6) include 12(13)EpOME and (E)-12,13-dihydroxy-11-methoxyoctadec- 9-enoic acid (Table 3). To our knowledge, this is the first report of those compounds with antibiofilm activity against $P$. aeruginosa PA14.

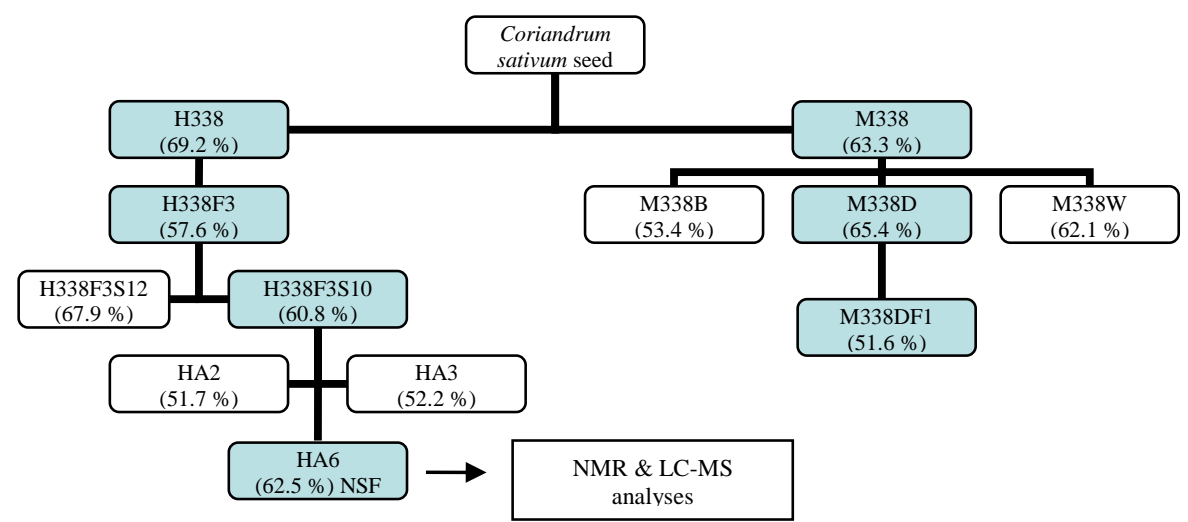

Figure 5 Fractionation scheme of the hexane $(\mathrm{H})$ ad methanol (M) extracts of Coriandrum sativum seeds (338)

Table $1{ }^{1} \mathrm{H}$ and ${ }^{13} \mathrm{C}$ NMR peak assignments for (HA6)

\begin{tabular}{llll}
\hline$\delta_{\mathrm{H}}(J \mathrm{~Hz})$ & $\begin{array}{l}\text { Proton } \\
\text { types }\end{array}$ & $\delta_{\mathrm{C}}(J \mathrm{~Hz})$ & Carbon types \\
\hline 0.86 & $-\mathrm{CH}_{3}$ & 15.51 & \\
1.26 & $-\left(\mathrm{CH}_{2}\right)_{\mathrm{n}}$ & 24.79 & $\mathrm{RCH}_{2-}$ \\
1.89 & $-\mathrm{CH}_{2-}$ & 25.17 & \\
$2.02 . .2 .04$ & $-\mathrm{CH}_{3}$ & 31.82 & \\
$2.11 . .2 .20$ & $-\mathrm{CH} 2-$ & 34.12 & $-\mathrm{CH}_{2-}$ \\
$2.68 . .2 .69$ & $-\mathrm{OH}$ & 181.95 & $\mathrm{C}=\mathrm{O}$ \\
$3.32 . .3 .35$ & $-\mathrm{CH}_{2-}$ & & \\
3.34 & $-\mathrm{CHOH}$ & & \\
$4.26 . .4 .28$ & $-\mathrm{CH}-$ & & \\
5.48 & $-\mathrm{CH}=\mathrm{CH}-$ & & \\
$8.52 . .8 .54$ & Ar-H & & \\
\hline
\end{tabular}

All spectra were recorded in $\mathrm{CD}_{3} \mathrm{OD}$ with $\delta_{\mathrm{H}}$ at $3.31 \mathrm{ppm}$ and $\delta_{\mathrm{C}}$ at $49.00 \mathrm{ppm}$ 
Table 2 Compounds identified in (HA6) using positive mode ESI-MS

\begin{tabular}{llllll}
\hline Compound & Formula & $\begin{array}{l}\text { Calculated } \\
\text { mass }\end{array}$ & $\begin{array}{l}\text { Theoretical } \\
\text { mass }\end{array}$ & $\begin{array}{l}\text { Mass } \\
\text { difference }\end{array}$ & $\begin{array}{l}\text { Error } \\
\text { (ppm) }\end{array}$ \\
\hline 2-hexylfuran $(\mathbf{C 2 2})$ & $\mathrm{C}_{10} \mathrm{H}_{16} \mathrm{O}$ & 152.1200851 & 152.1201152 & -0.0000301 & -0.1981329 \\
Linalool oxide $(\mathbf{C 2})$ & $\mathrm{C}_{10} \mathrm{H}_{18} \mathrm{O}_{2}$ & 170.1306442 & 170.1306799 & -0.0000357 & -0.2099563 \\
Linalool oxide $(\mathbf{C 2 3})$ & $\mathrm{C}_{10} \mathrm{H}_{18} \mathrm{O}_{2}$ & 170.1307357 & 170.1306799 & 0.0000558 & 0.3278656 \\
10-undecenal $(\mathbf{C 2 4})$ & $\mathrm{C}_{11} \mathrm{H}_{20} \mathrm{O}$ & 168.1514114 & 168.1514154 & -0.0000040 & -0.0237881 \\
4-Ethylcamphor $(\mathbf{C 2 5})$ & $\mathrm{C}_{12} \mathrm{H}_{20} \mathrm{O}$ & 180.1513809 & 180.1514154 & -0.0000345 & -0.1915056 \\
Dodecenal $(\mathbf{C 2 6})$ & $\mathrm{C}_{12} \mathrm{H}_{24} \mathrm{O}$ & 184.1827072 & 184.1827156 & -0.0000084 & -0.0453897 \\
Caryophyllene oxide $(\mathbf{C 2 7})$ & $\mathrm{C}_{15} \mathrm{H}_{24} \mathrm{O}$ & 220.1826919 & 220.1827156 & -0.0000237 & -0.1074562 \\
\hline
\end{tabular}

Table 3 Compounds identified in (HA6) using negative mode ESI-MS

\begin{tabular}{llllll}
\hline Compound & Formula & $\begin{array}{l}\text { Calculated } \\
\text { mass }\end{array}$ & $\begin{array}{l}\text { Theoretical } \\
\text { mass }\end{array}$ & $\begin{array}{l}\text { Mass } \\
\text { difference }\end{array}$ & $\begin{array}{l}\text { Error } \\
(\mathrm{ppm})\end{array}$ \\
\hline 12(13)-EpOME (C28) & $\mathrm{C}_{18} \mathrm{H}_{32} \mathrm{O}_{3}$ & 296.2351395 & 296.2351438 & -0.0000043 & -0.0145254 \\
$\begin{array}{l}\text { (E)-12,13-dihydroxy-11- } \\
\text { methoxyoctadec- 9-enoic acid } \\
\text { (C29) }\end{array}$ & $\mathrm{C}_{19} \mathrm{H}_{36} \mathrm{O}_{5}$ & 344.2562729 & 344.2562730 & -0.0000001 & -0.0002326 \\
\hline
\end{tabular}

\section{CONCLUSION}

The effect of plant extracts in destabilising and inhibiting biofilms is a promising approach for the discovery of new antibiofilm hits and drug leads. This study led to the identification of potential bioactive compounds from the selected plant as natural antibiofilm agents against $P$. aeruginosa PA14. The compounds were mixture of alkanes from Ribes nigrum leaf, mixture of ursolic acid and oleanolic acid from Sambucus nigra flowers and mixture of oxygenated monoterpenes, carboxylic acid, carboxylate, tetraone, glycerol, carbohydrates and fatty acids from Coriandrum sativum seeds. More in-depth study therefore could investigate the mechanism beneath the inhibition of $P$. aeruginosa PA14 biofilm using these compounds in the near future. Notably more researches needed to be done before the compounds can be considered as potent antibiofilm agents. Thus, in future studies, antibiofilm activity of those compounds against positive control (gentamicin) and other pure natural products either alone or in mixedratios will be investigated. Others, such as activity of those compounds against other biofilms of other Pseudomonas strains also tend to be evaluated. Results discovered from these findings therefore will be presented and discussed separately in the next publications.

\section{ACKNOWLEDGEMENT}

The author would like to thank MARDI for the financial support in conducting this research at Strathclyde Institute of Pharmacy and Biomedical Sciences, University of Strathclyde, Glasgow, UK.

\section{REFERENCES}

[1] Suzutani, T., Ogasawara, M., Yoshida, I., Azuma, M. and Knox, Y. M. (2003). Anti-herpesvirus activity of an extract of Ribes nigrum L. Phytotherapy Research, 17, 609-13.

[2] Budzynska, A., Wieckowska-Szakiel, M., Sadowska, B., Kalemba, D. and Rozalska, B. (2011). Antibiofilm activity of selected plant essential oils and their major components. Polish Journal of Microbiology, 60, 3541. 
[3] Beutler, J. A. (2009). Natural products as a foundation for drug discovery. Current Protocols in Pharmacology, 46, 9111.

[4] Church, D., Elsayed, S., Reid, O., Winston, B. and Lindsay, R. (2006). Burn wound infections. Clinical Microbiology Reviews, 19, 403-34.

[5] Lang, G. and Buchbauer, G. (2012). A review on recent research results (2008-2010) on essential oils as antimicrobials and antifungals. A review Flavour and Fragrance Journal, 27, 13-39.

[6] Suzutani, T., Ogasawara, M., Yoshida, I., Azuma, M. and Knox, Y. M. (2003). Anti-herpesvirus activity of an extract of Ribes nigrum L. Phytotherapy Research, 17, 609-13.

[7] Quave, C. L., Estevez-Carmona, M., Compadre, C. M., Hobby, G., Hendrickson, H., Beenken, K. E. and Smeltzer, M. S. (2012). Ellagic acid derivatives from Rubus ulmifolius inhibit Staphylococcus aureus biofilm formation and improve response to antibiotics. PLoS One, 7, e28737.

[8] Jiang, X., Yu, P., Jiang, J., Zhang, Z., Wang, Z., Yang, Z., Tian, Z., Wright, S. C., Larrick, J. W. and Wang, Y. (2009). Synthesis and evaluation of antibacterial activities of andrographolide analogues. European Journal of Medicinal Chemistry, 44, 2936-43.

[9] Lee, D. G., Urbach, J. M., Wu, G., Liberati, N. T., Feinbaum, R. L., Miyata, S., Diggins, L. T., He, J., Saucier, M., Deziel, E., Friedman, L., Li, L., Grills, G., Montgomery, K., Kucherlapati, R., Rahme, L. G. and Ausubel, F. M. (2006). Genomic analysis reveals that Pseudomonas aeruginosa virulence is combinatorial. Genome Biology, 7, R90.

[10] Adnan, H. (2015). Studies on the activity of selected plants against biofilms of Pseudomonas aeruginosa strain PA14. (Ph.D. thesis, University of Strathclyde, Glasgow, UK). Retrieved from http://ethos.bl.uk (EThOS ID:uk.bl.ethos668862)

[11] Duman, A. D., Telci, I., Dayisoylu, K. S., Digrak, M., Demirtas, I. and Alma, M. H. (2010). Evaluation of bioactivity of linalool-rich essential oils from Ocimum basilucum and Coriandrum sativum varieties. Natural Product Communications, 5, 969-74.

[12] Awen, B. Z. S., Unnithan, C. R., Ravi, S., Kermagy, A., Sasikumar, J. M., Khrbash, A. S. and Ekreem, W. L. (2011). Essential oils of Retama raetam from Libya: Chemical composition and antimicrobial activity. Natural Product Research, 25, 927-33.

[13] Rattanachaikunsopon, P. and Phumkhachorn, P. (2010). Potential of coriander (Coriandrum sativum) oil as a natural antimicrobial compound in controlling Campylobacter jejuni in raw meat. Bioscience, Biotechnology and Biochemistry, 74, 31-5.

[14] Lixandru, B. E., Dracea, N. O., Dragomirescu, C. C., Dragulescu, E. C., Coldea, I. L., Anton, L., Dobre, E., Rovinaru, C. and Codita, I. (2010). Antimicrobial activity of plant essential oils against bacterial and fungal species involved in food poisoning and/or food decay. Roumanian Archives of Microbiology and Immunology, 69, 224-30.

[15] Gellatly, S. L. and Hancock, R. E. W. (2013). Pseudomonas aeruginosa: new insights into pathogenesis and host defenses. Pathogens and Disease, 67, 159-73.

[16] Witkowska, A. M., Hickey, D. K., Alonso-Gomez, M. and Wilkinson, M. G. (2011). The microbiological quality of commercial herb and spice preparations used in the formulation of a chicken supreme ready meal and microbial survival following a simulated industrial heating process. Food Control, 22, 616-25.

[17] Silva, F., Ferreira, S., Queiroz, J. A. and Domingues, F. C. (2011). Coriander (Coriandrum sativum L.) essential oil: its antibacterial activity and mode of action evaluated by flow cytometry. Journal of Medicinal Microbiology, 60, 1479-86.

[18] Furletti, V. F., Teixeira, I. P., Obando-Pereda, G., Mardegan, R. C., Sartoratto, A., Figueira, G. M., Duarte, R. M. T., Rehder, V. L. G., Duarte, M. C. T., \#246 and Fling, J. F. (2011). Action of Coriandrum sativum L. essential oil upon oral Candida albicans biofilm formation. Evidence-Based Complementary and Alternative Medicine, 2011. 